\title{
ON THE CHERN CLASSES OF REPRESENTATIONS OF FINITE GROUPS $\left({ }^{1}\right)$
}

\author{
BY \\ LEONARD EVENS
}

1. Introduction. Let $G$ be a finite group. Following Atiyah [1] and others, we may define the Chern classes $c_{i}(\rho) \in H^{2 i}(G, Z)$ for each unitary representation $\rho$ of $G$. In order to compute the Chern classes we may restrict $\rho$ to the Sylow subgroups of $G$ for the primes dividing its order and use the Sylow subgroup argument in cohomology. Thus, in principle, it is enough to assume that $G$ is a $p$-group. Blichfeldt's theorem $[8, \S 258$, Theorem XII] says that every irreducible representation of a $p$-group is induced from a one-dimensional representation of some subgroup. It is the purpose of this paper to investigate the Chern classes of such a representation.

Let $H$ be a subgroup of $G$ and $\lambda \in \hat{H}=H^{2}(H, Z)$ a one-dimensional representation of $H$. In [5], I defined a mapping $\mathscr{N}_{H \rightarrow G}: H^{*}(H, Z) \rightarrow H^{*}(G ; Z)$ which is a "multiplicative" generalization of transfer. Viewing $\lambda$ as a cohomology class, we may write $\mathscr{N}_{H \rightarrow G}(1+\lambda)=\sum \mathscr{N}_{i}(\lambda)$ where $\mathscr{N}_{i}(\lambda)$ is of degree $2 i, \mathscr{N}_{1}$ is the transfer, and $\mathscr{N}_{(G: H)}(\lambda)=\mathscr{N}_{H \rightarrow G}(\lambda)$.

It is shown here that, in a certain sense, $\mathscr{N}_{i}(\lambda)$ is the leading term of $c_{i}(\rho)$ where $\rho=\operatorname{ind}_{H \rightarrow G} \lambda$ is the representation of $G$ induced from $\lambda$. In fact, for the highest Chern class, we have $c_{(G: H)}($ ind $\lambda)=\mathscr{N}_{H \rightarrow G}(\lambda)$. Moreover, the other terms are determined in that it is shown how to compute them, in principle only, by a procedure entirely within the algebraic theory of cohomology of groups.

One sees quite easily that the basic problem is to compute the Chern classes of the inclusion representation of the normalizer of a maximal torus in the unitary group. In $\$ 3$, this problem is solved explicitly in terms of the Chern classes of inclusion representations of tori (elementary symmetric functions), and the Chern classes of the natural representations of the symmetric groups by permutation matrices.

Finally, the results, are applied to computing the map $\mathscr{N}$ and Chern classes in special cases.

For the facts concerning Chern classes and the map $\mathscr{N}, I$ refer the reader respectively to $[1]$ and $[5]$.

2. Topological preliminaries. Let $G$ be a compact Lie group. There exists a principal $G$-bundle with contractible total space $E_{G}$ (the universal space), and the base space $B_{G}$ of such a bundle is called a classifying space for $G$.

Received by the editors March 22, 1963 and, in revised form, July 2y, 1963.

( ${ }^{1}$ Partially supported by National Science Foundation grants G-19137 and G-24943. 
These spaces may be chosen within a convenient category ([6], $[2, \mathrm{I}, \S 1])$, so that, given $\varphi: G \rightarrow G^{\prime}$, a homomorphism of compact Lie groups, there is a map $F: E_{G} \rightarrow E_{G}$ of corresponding universal spaces, consistent with $\varphi$ (i.e. $F(g e)=\varphi(g) F(e)$, for $\left.g \in G, e \in E_{G}\right)$, which induces a map $f: B_{G} \rightarrow B_{G}$. Moreover, different $F$ 's induce homotopic $f$ 's.

Let $S$ be a subgroup of the symmetric group $\mathscr{S}_{k}$ on $k$ symbols. We may choose for $E_{S}$ a countable $\mathrm{CW}$ complex such that each cell is carried into a disjoint cell by each element of $S$. (In fact, if we choose such a space for $\mathscr{S}_{k}$ it will do for every subgroup; this fact will be useful below.) Let $X$ be a CW complex with finitely many cells in each dimension (e.g. $B_{H}, H$ a compact Lie group). $S$ acts on $X^{k}$ by permuting the factors, and it acts diagonally on $E_{S} \times X^{k}$. (All actions of one thing on another are to be on the left.) Moreover, the elements of $S$ carry to cells of $E_{S} \times X^{k}$ into disjoint cells so that the orbit space $E_{S} \times_{S} X^{k}$ becomes a $\mathrm{CW}$ complex by projecting the cells of $E_{S} \times X^{k}$. Thus, there are natural isomorphisms of chain or cochain complexes

$$
\begin{aligned}
& C_{*}\left(E_{S} \times{ }_{S} X^{k}\right) \cong C_{*}\left(E_{S}\right) \otimes_{S} C_{*}(X)^{k}, \\
& C_{*}\left(E_{S} \times X_{S} X^{k}\right) \cong \operatorname{Hom}_{S}\left(C_{*}\left(E_{S}\right), C^{*}(X)^{k}\right) .
\end{aligned}
$$

(Whenever a coefficient group is suppressed, assume it is $Z$.)

Assume next that $H^{*}(X)$ is free. In what follows, I imitate Nakaoka $[7, \S 3]$. Consider the obvious homomorphisms of the group of cocycles

$$
H^{*}(X) \stackrel{j}{\longleftarrow} Z^{*}(X) \stackrel{i}{\longrightarrow} C^{*}(X) .
$$

Since $H^{*}(X)$ is free, there is an arrow $h: H^{*}(X) \rightarrow Z^{*}(X)$ such that $j h$ $=1$. Also, with zero differentiations, $h$ is a map of cochain complexes. Set $q=i h: H^{*}(X) \rightarrow C^{*}(X)$. As in Nakaoka [7], $q$ is a cochain equivalence, and hence $Q=\operatorname{Hom}_{S}\left(C_{*}\left(E_{S}\right), q^{k}\right)$ is a cochain equivalence. Thus

$$
Q^{*}: H^{*}\left(S, H^{*}(X)^{k}\right) \longrightarrow H^{*}\left(E_{S} \times{ }_{S} X^{k}\right)
$$

is an isomorphism. $Q^{*}$ is certainly natural with respect to inclusions of one subgroup of $\mathscr{S}_{k}$ in another. Let $S^{\prime}<S$ be two such subgroups. The finite covering $E_{S} \times_{S^{\prime}} X^{k} \rightarrow E_{S} \times{ }_{S} X^{k}$ gives rise to a transfer map

$$
\operatorname{tr}\left[S^{\prime} \rightarrow S\right]: H^{*}\left(E_{S} \times_{S^{\prime}} X^{k}\right) \rightarrow H^{*}\left(E_{S} \times{ }_{S} X^{k}\right),
$$

which is described as follows on the cochain level. Let $\beta_{1}, \beta_{2}, \ldots, \beta_{\left(S: S^{\prime}\right)}$ be a complete set of representatives of the left cosets of $S^{\prime}$ in $S$. If

$$
f \in \operatorname{Hom}_{S^{\prime}}\left(C_{*}\left(E_{S}\right), C^{*}(X)^{k}\right)
$$

represents $\alpha$, then $\operatorname{tr} \alpha$ is represented by $\sum \beta_{i} f \in \operatorname{Hom}_{S}\left(C_{*}(X), C^{*}(X)^{k}\right)$, where $\left(\beta_{i} f\right)(w)=\beta_{i}\left(f\left(\beta_{i}^{-1} w\right)\right)$. I claim that $Q^{*}$ is consistent with transfer, 
i.e. the isomorphism $Q^{*}$ carries this transfer map into the ordinary

$$
\operatorname{tr}\left[S^{\prime} \rightarrow S\right]: H^{*}\left(S^{\prime}, H^{*}(X)^{k}\right) \longrightarrow H^{*}\left(S, H^{*}(X)^{k}\right)
$$

of group cohomology. This is true because, on the cochain level, $Q$ certainly commutes with transfer. Finally, $Q^{*}$ is natural with respect to maps $f: X_{1} \rightarrow X_{2}$ of CW complexes. For, if $H^{*}\left(X_{1}\right)$ and $H^{*}\left(X_{2}\right)$ are both free, choose $q_{i}: H^{*}\left(X_{i}\right) \rightarrow C^{*}\left(X_{i}\right)$ as above. Then, $f \cdot q_{1}$ and $q_{2} \cdot H^{*}(f)$ induce the same homomorphism in cohomology; since $H^{*}\left(X_{1}\right)$ is free, it follows that these two maps are cochain homotopic. Thus, by the lemma of Steenrod [ $7, \S 3]$, the diagram

$$
\begin{gathered}
H^{*}\left(S, H^{*}\left(X_{1}\right)^{k}\right) \stackrel{Q_{1}^{*}}{\longrightarrow} H^{*}\left(E_{S} \times_{S} X_{1}^{k}\right) \\
H^{*}\left(1_{S}, H^{*}(f)^{k}\right) \mid \\
H^{*}\left(S, H^{*}\left(X_{2}\right)^{k}\right) \stackrel{Q_{2}^{*}}{\longrightarrow} H^{*}\left(E_{S} \times_{S} X_{2}^{k}\right)
\end{gathered}
$$

commutes.

$Q^{*}$ is also multiplicative - the product on the left being defined in the obvious way $[5, \S 4,4]$. To see this proceed as follows. Since $S$ is finite there is a cellular map $\Delta_{S}: E_{S} \rightarrow E_{S} \times E_{S}$ which is consistent with the diagonal monomorphism $\delta: S \rightarrow S \times S$ and which is homotopic to the diagonal map of $E_{S}$ into $E_{S} \times E_{S}$ by a homotopy consistent with $\delta$. Let $\Delta_{X}: X \rightarrow X \times X$ be a cellular map homotopic to the diagonal map. Define $\Delta$ from the commutative diagram

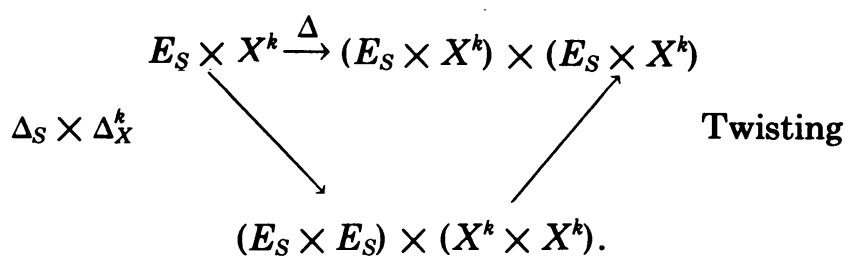

$\Delta$ is a cellular map consistent with $\delta$ and homotopic to the diagonal map by a homotopy consistent with $\delta$. Thus, $\Delta$ induces a cellular map $\bar{\Delta}$ : $E_{S} \times_{S} X^{k} \rightarrow\left(E_{S} \times_{S} X^{k}\right)^{2}$ homotopic to the diagonal. Finally, under the isomorphism (1), the chain map induced by $\bar{\Delta}$ corresponds to $\left(\Delta_{S}\right)_{*} \otimes_{S}\left(\Delta_{X}\right)_{*}^{k}$ followed by twisting. Since this chain map induces the product referred to above, we are reduced essentially to the situation of Nakaoka. See $[7, \S 3]$ for details.

Finally, suppose $S<\mathscr{S}_{k}$ and $H$ is a compact Lie group. Denote by $S \int H$ the semidirect product $S \cdot H^{k}$ where $S$ acts on $H^{k}$ by permuting the factors. (This wreath product is clearly functorial in $S$ and $H$. If $\theta$ : $H \rightarrow H^{\prime}$ is a homomorphism of groups, we shall use

$$
1 \int \theta: S \int H \rightarrow S \int H^{\prime}
$$


for the induced homomorphism. See [5] for details.) If $E_{H}$ is a universal space for $H$, then $E_{S} \times E_{H}^{k}$ is a universal space for $S \boldsymbol{S} H$ where $H^{k}$ acts componentwise on $E_{H}^{k}$ and $S$ acts diagonally as above. Factoring first by $H^{k}$ and then by $S$, we see that $E_{S} \times{ }_{S} B_{H}^{k}$ is a classifying space for $S \int H$, and we may apply the above analysis.

In particular, under $Q^{*}$, the following homomorphisms of groups induce the indicated homomorphisms of cohomology:

(i) $H^{k}=\{1\} \int H<S \int H$,

$$
H^{*}\left(S, H^{*}\left(B_{H}\right)^{k}\right) \stackrel{\text { projection }}{\longrightarrow}\left(H^{*}\left(B_{H}\right)^{k}\right)^{S} \subset H^{*}\left(B_{H^{k}}\right) ;
$$

(ii) $S \int H \rightarrow S \int\{1\}=S$,

$$
H^{*}(S, Z)=H^{*}\left(S, H^{0}\left(B_{H}\right)^{k}\right) \subset H^{*}\left(S, H^{*}\left(B_{H}\right)^{k}\right) \text {; }
$$

(iii) $S=S \int\{1\}<S \int H$,

$$
H^{*}\left(S, H^{*}\left(B_{H}\right)^{k}\right) \stackrel{\text { projection }}{\longrightarrow} H^{*}(S, Z) .
$$

(In (i) the naturality with respect to $S$ is used, and in (ii) and (iii) the maps involved are between $B_{H}$ and a point.)

Since the methods used here will be those of the cohomology theory of groups, we shall adopt the terminology of that theory in the more general context. A homomorphism induced by inclusion of one group in another will be called restriction (abbreviated "res"), one induced by an epimorphism will be called inflation ("inf"). Also, where it is necessary to indicate the source and target groups, these will be contained in brackets following the generic symbol for the homomorphism.

3. Chern classes. Let $T$ denote the circle group. According to $\$ 2$, the cohomology ring of the classifying space of $\mathscr{S}_{k} \int T$ is given by $H^{*}$ $\left(\mathscr{S}_{k}, Z\left[X_{1}, X_{2}, \cdots, X_{k}\right]\right)$ where the $X_{j}$ are of degree 2. For convenience, write $\mathscr{P}_{j}^{k}$ for the homogeneous çomponent of $Z\left[X_{1}, \cdots, X_{k}\right]=H^{*}\left(B_{T^{k}}, Z\right)$ of degree $2 j$. Let $\pi_{k}: \mathscr{S}_{k} \rightarrow U_{k}$ be the "natural" representation of $\mathscr{S}_{k}$ by permutation matrices, let $T^{k}$ be identified with the subgroup of $U_{k}$ consisting of diagonal matrices. For the moment, identify $\mathscr{S}_{k}$ with the group of permutation matrices via $\pi_{k}$. Then, $\mathscr{S}_{k}$ normalizes $T^{k}$, and the subgroup of $U_{k}$ they generate is the normalizer $N_{k}$ of a maximal torus. Clearly, $N_{k}$ is the semidirect product of the subgroups $\mathscr{S}_{k}$ and $T^{k}$, and it may be identified with $\mathscr{S}_{k} \int T$. We wish to determine the restrictions of the Chern classes in $H^{*}\left(B_{U_{k}}, Z\right)$ to $H^{*}\left(B_{N_{k}}, Z\right)$; we denote these by $C_{n}^{k} \in H^{2 n}\left(B_{N_{k}}, Z\right)$. Because of the decomposition of $\S 2$, we may write

$$
C_{n}^{k}=\sum_{i+j=n} C_{i, j}^{k}
$$

where $C_{i, j}^{k} \in H^{2 i}\left(\mathscr{S}_{k}, \mathscr{P}_{j}^{k}\right)$. Since, upon restriction to $T^{k}$, the Chern classes become the elementary symmetric functions $\phi_{n}^{k}\left(X_{1}, \cdots, X_{k}\right)$, we have 


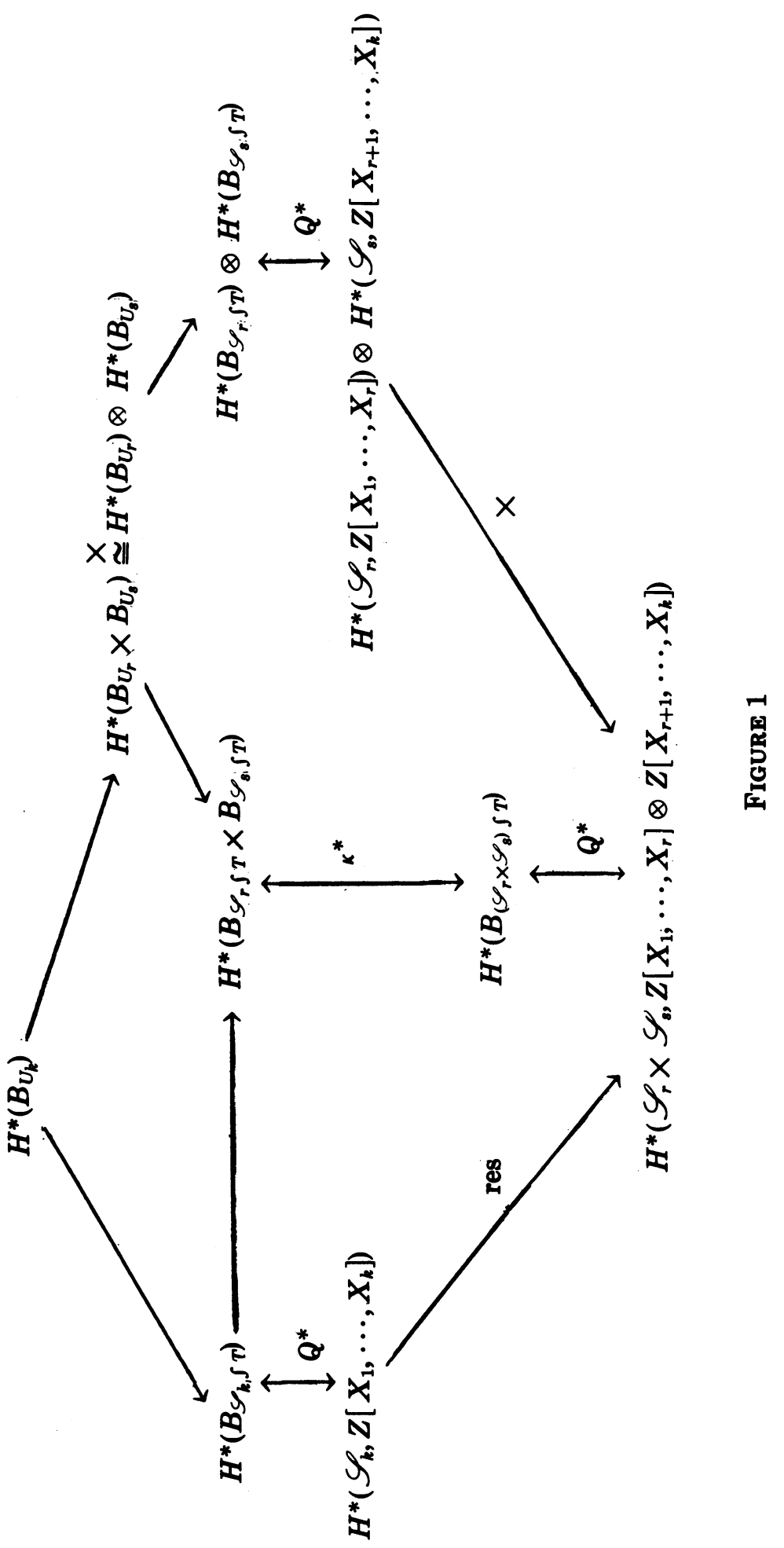




$$
C_{0, n}^{k}=\phi_{n}^{k} \in H^{0}\left(\mathscr{S}_{k}, \mathscr{P}_{n}^{k}\right) .
$$

Similarly,

$$
C_{n, 0}^{k}=\inf \left[\mathscr{S} \rightarrow \mathscr{S} \int T\right] \operatorname{res}[\mathscr{S} \mathcal{S} \Gamma \rightarrow \mathscr{S}]\left(C_{n}^{k}\right),
$$

or, under the above identifications,

$$
C_{n, 0}^{k}=c_{n}\left(\pi_{k}\right) \in H^{2 n}\left(\mathscr{S}_{k}, Z\right)
$$

write $\gamma_{n}^{k}=c_{n}\left(\pi_{k}\right)$.

Let $K=\left\{X_{1}, X_{2}, \cdots, X_{k}\right\}$, and suppose $K=R \cup S$ is a partition of $K$. (Consider the partitions $R \cup S$ and $S \cup R$ different!) Write $r$ for the number of elements in $R$, etc. We shall be particularly interested in partitions for which $R$ contains the first $r$ consecutive $X_{i}$ and $S$ the next $s$ consecutive $X_{i}$; we shall call such a partition a standard partition. Let $\mathscr{S}_{r}=\mathscr{S}(R)$ denote the group of permutations of $R$, similarly for $S$; then, $\mathscr{S}_{r} \times \mathscr{S}_{s}$ may be naturally identified with a subgroup of $\mathscr{S}_{k}$. Also, the $k$-fold wreath product $\left(\mathscr{S}_{r} \times \mathscr{S}_{s}\right) \int_{k} T$ becomes a subgroup of $\mathscr{S}_{k} \int_{k} T$, and we have a natural isomorphism

$$
\kappa:\left(\mathscr{S}_{r} \int_{r} T\right) \times\left(\mathscr{S}_{s} \int_{s} T\right) \cong\left(\mathscr{S}_{r} \times \mathscr{S}_{s}\right) \int_{k} T .
$$

Denote by $X$ the external product of cohomology classes. Referring to Figure 1 (in which the unlabelled arrows are appropriate restrictions), we see that

$$
\operatorname{res}\left(C_{i, j}^{k}\right)=\sum_{i_{1}+i_{2}=i, j_{1}+j_{2}=j} C_{i_{1}, j_{1}}^{r} \times C_{i_{2}, j_{2}}^{s}
$$

Theorem 1.

$$
C_{i, j}^{k}=\operatorname{tr}\left[\left(\mathscr{S}_{k-j} \times \mathscr{S}_{j}\right) \int T \rightarrow \mathscr{S}_{k} \int T\right]\left(\gamma_{i}^{k-j} \times \phi_{j}^{j}\right) .
$$

Proof. To each monomial in the $X_{i}$ we can associate a unique partition $K=K_{0} \cup K_{1} \cup K_{2} \cup \ldots$ (distinguishing again between different orderings of the subsets); namely, let $K_{t}$ consist of all $X_{i}$ with exponent $t$ in the monomial. Clearly, each partition comes from a unique monomial. If $k_{t}$ is the cardinality of $K_{t}$, then $\sum k_{t}=k$, where all but a finite number of $k_{t}$ are zero. (In such a sum, sum always from 0 to $\infty$.)

Given such a partition $K=K_{0} \cup K_{1} \cup \cdots$, denote by $\left[K_{0}, K_{1}, \cdots\right]$ the $\mathscr{S}_{k}$-submodule of $Z\left[X_{1}, \ldots, X_{k}\right]$ generated by the monomial associated with the partition. Then, $\mathscr{P}_{j}^{k}$ is the direct sum of all $\left[K_{0}, K_{1}, \ldots\right]$ for which $\sum t k_{t}=j$, and

$$
H^{*}\left(\mathscr{S}_{k}, \mathscr{P}_{j}^{k}\right)=\sum H^{*}\left(\mathscr{S}_{k},\left[K_{0}, K_{1}, \cdots\right]\right) .
$$

Let $\left(C_{i, j}^{k} ; K_{0}, K_{1}, \ldots\right)$ be the component of $C_{i, j}^{k}$ in $H^{2 i}\left(\mathscr{S}_{k},\left[K_{0}, K_{1}, \ldots\right]\right)$; we shall compute these quantities.

Since within each transitivity set, there is always a monomial with exponents nondecreasing (with the $X_{i}$ written in the natural order), it will 
certainly be sufficient to consider only standard partitions when studying the structure of the modules defined above. As before, given a (standard) partition $K=K_{0} \cup K_{1} \cup \cdots, \mathscr{A}\left(K_{0}\right) \times \mathscr{A}\left(K_{1}\right) \times \cdots$ may be identified with a subgroup of $\mathscr{S}_{k}$, in fact, the subgroup fixing the associated (standard) monomial. Moreover, $\left[K_{0}, K_{1}, \ldots\right]$ is simply the $\mathscr{S}_{k}$-module induced from the trivial $\left(\mathscr{S}\left(K_{0}\right) \times \mathscr{Y}\left(K_{1}\right) \times \cdots\right)$-module $Z$. We shall decompose this induced module as a module over the subgroup $\mathscr{S}\left(K_{0}\right) \times \mathscr{A}\left(K_{1}\right) \times \cdots$. Let $K_{t}=K_{t, 0} \cup K_{t, 1} \cup \ldots$ be a standard partition of $K_{t}$ for each $t=0$, $1, \ldots$ such that $k_{s}=\sum_{t} k_{t, s}$ for each $s=0,1, \ldots$ (If we set $\bar{K}_{s}=K_{0,8} \cup K_{1, s}$ $\cup \cdots$, then the partition $K=\bar{K}_{0} \cup \bar{K}_{1} \cup \cdots$ corresponds to a monomial with the same set of exponents as the original standard monomial and the variables permuted so that within each $K_{t}$ the exponents are nondecreasing. Also, each such monomial gives rise to a unique such subpartition.) Then $\left[K_{0}, K_{1}, \ldots\right]$ decomposes into the direct sum of the $\left(\mathscr{S}\left(K_{0}\right) \times \mathscr{S}\left(K_{1}\right) \times \cdots\right)$-modules

$$
\sum\left[K_{0,0}, K_{0,1}, \cdots\right] \otimes\left[K_{1,0}, K_{1,1}, \cdots\right] \otimes \cdots,
$$

where the sum is taken over all subpartitions of the above kind. To see this, simply notice that any monomial with the same set of exponents as the original standard monomial may be transformed by an element of $\mathscr{S}\left(K_{0}\right) \times \mathscr{S}\left(K_{1}\right) \times \ldots$ into one for which within each $K_{t}$ the exponents are nondecreasing. As the $\left(\mathscr{A}\left(K_{0}\right) \times \mathscr{S}\left(K_{1}\right) \times \cdots\right)$-module generated by such a monomial is a tensor product of the above form, the decomposition (3) is clear. (Needless to say, the above argument is only a rederiving of the familiar double coset law in this specific case.)

If follows from formulas (2) and (3) that the restriction of $\left(C_{i, j}^{k} ; K_{0}, K_{1}, \ldots\right)$ to $\left(\mathscr{A}\left(K_{0}\right) \times \mathscr{S}\left(K_{1}\right) \times \ldots\right) \int T$ is given by

$$
\sum\left(C_{i_{0}, j_{0}}^{k_{0}} ; K_{0,0}, K_{0,1}, \cdots\right) \times\left(C_{i_{1}, j_{1}}^{k_{1}} ; K_{1,0}, K_{1,1}, \cdots\right) \times \cdots
$$

where the sum is taken over all subpartitions of the above kind and all indices such that

$$
\sum_{t} k_{s, t}=k_{s}, \quad \sum_{s} k_{s, r}=k_{r}, \quad \sum_{t} t k_{s, t}=j_{s}, \quad \text { for } r, s=0,1, \cdots,
$$

and

$$
\sum_{s} j_{s}=j, \quad \sum_{s} i_{s}=i
$$

The sum is trivial unless $0 \leqq k_{0}=k-j, k_{1}=j \leqq k$, and $k_{r}=0$ for $r>1$; i.e. unless $K=K_{0} \cup K_{1}$. In fact, $C_{i_{s}, j_{s}}^{k_{s}}=0$ unless $i_{s}+j_{s} \leqq k_{s}$. But, by (5), this entails $\sum_{t} t k_{s, t} \leqq \sum_{t} k_{s, t}$ or $k_{s, t}=0$ for $t>1$. Thus $k_{r}=\sum_{s} k_{s, r}=0$ for $r>1$. The following fact, which is an easy consequence of Shapiro's Lemma [3, Chapter X, Proposition 7.4], Allows us to conclude that $\left(C_{i, j}^{k} ; K_{0}, K_{1}, \cdots\right)=0$ unless $K=K_{0} \cup K_{1}$. 
Lemma. Let $G$ be a group, $H$ a subgroup of finite index, and $M$ a G-module induced from an $\mathrm{H}$-module. Then

$$
\operatorname{res}[H \rightarrow G]: H^{*}(G, M) \rightarrow H^{*}(H, M)
$$

is an injection.

To complete the proof of the theorem, we consider the case $K=K_{0} \cup K_{1}$. (Drop ",..." from the notation now.) As above, the restriction of $C_{i, j}^{k}$ $=\left(C_{i, j}^{k} ; K_{0}, K_{1}\right)$ to $\left(\mathscr{A}\left(K_{0}\right) \times \mathscr{S}\left(K_{1}\right)\right) \delta T$ is

$$
\sum\left(C_{i_{0}, j_{0}}^{k_{0}} ; K_{0,0}, K_{0,1}\right) \times\left(C_{i_{1}, j_{1}}^{k_{1}} ; K_{1,0}, K_{1,1}\right)
$$

where $i_{0}+i_{1}=i, \quad j_{0}+j_{1}=j, \quad k_{0,0}=k-j-j_{0}, \quad k_{0,1}=j_{0}, k_{1,0}=j-j_{1}, k_{1,1}$ $=j_{1}$. Since the desired formula is certainly true for $k=1$, we may proceed by induction. The above sum then becomes

$$
\begin{gathered}
\sum \operatorname{tr}\left[\mathscr{S}\left(K_{0,0}\right) \times \mathscr{A}\left(K_{0,1}\right) \times \mathscr{A}\left(K_{1,0}\right) \times \mathscr{S}\left(K_{1,1}\right) \rightarrow \mathscr{S}\left(K_{0}\right) \times \mathscr{S}\left(K_{1}\right)\right] \\
\left\{\left(\gamma_{i_{0}, 0}^{k_{0}} \times \phi_{j_{0}}^{j_{0}}\right) \times\left(\gamma_{i_{1}}^{k_{1,0}} \times \phi_{j_{1}}^{j_{1}}\right)\right\} .
\end{gathered}
$$

(Use the formula $\operatorname{tr}\left[H \times H^{\prime} \rightarrow G \times G^{\prime}\right]=\operatorname{tr}[H \rightarrow G] \times \operatorname{tr}\left[H^{\prime} \rightarrow G^{\prime}\right]$. Also notice that it is legitimate to drop " $T$ " because of the results of $\$ 2$.) Consider next

$$
\operatorname{res}\left[\mathscr{S}(K) \rightarrow \mathscr{N}\left(K_{0}\right) \times \mathscr{N}\left(K_{1}\right)\right] \operatorname{tr}\left[\mathscr{N}\left(K_{0}\right) \times \mathscr{S}\left(K_{1}\right) \rightarrow \mathscr{S}(K)\right]\left(\gamma_{i}^{k_{0}} \times \phi_{j}^{j}\right)
$$

If we can show that this quantity is also given by (6), the theorem will follow by the lemma above.

Let $\tau$ be the permutation

$$
\left(\begin{array}{l}
K_{0,0}, K_{0,1}, K_{1,0}, K_{1,1} \\
K_{0,0}, K_{1,0}, K_{0,1}, K_{1,1}
\end{array}\right)
$$

and let $\bar{K}_{t}=\tau\left(K_{t}\right)$. For $\alpha \in H^{*}\left(\mathscr{A}\left(K_{0}\right) \times \mathscr{S}\left(K_{1}\right), A\right)$, let

$$
\bar{\alpha}=c_{r}(\alpha) \in H^{*}\left(\mathscr{S}\left(\bar{K}_{0}\right) \times \mathscr{S}\left(\bar{K}_{1}\right), A\right)
$$

(where $A$ is any $\mathscr{S}_{k}$-module). Then the composite map above applied to $\alpha$ yields a sum of terms of the form

$$
\text { tr res }\left[\mathscr{A}\left(\bar{K}_{0}\right) \times \mathscr{S}\left(\bar{K}_{1}\right) \rightarrow \mathscr{S}\left(K_{0,0}\right) \times \mathscr{S}\left(K_{0,1}\right) \times \mathscr{S}\left(K_{1,0}\right) \times \mathscr{A}\left(K_{1,1}\right)\right](\bar{\alpha})
$$

where the summation is extended over all subpartitions of the allowed kind. This is just the double coset rule for resotr [3, Chapter XII, Proposition 9.1]. It is fairly easy to see that we are in fact summing over a complete set of representatives of the double cosets

$$
\left(\mathscr{S}\left(K_{0}\right) \times \mathscr{S}\left(K_{1}\right)\right) \rho\left(\mathscr{S}\left(K_{0}\right) \times \mathscr{S}\left(K_{1}\right)\right) .
$$

(We have already made use of this implicitly above.) For, any permutation may be modified on the right by an element of $\mathscr{A}\left(K_{0}\right) \times \mathscr{A}\left(K_{1}\right)$ so as to be order-preserving when restricted to each $K_{t}$, and modified then on the left so as to carry the standard monomial into one with exponents 
nondecreasing within each $K_{t}$. (Strictly speaking, the above formula should include a sign since viewing $\mathscr{S}\left(\bar{K}_{0}\right) \times \mathscr{S}\left(\bar{K}_{1}\right)$ as a subgroup of $\mathscr{S}_{k}$ involves twisting; however, since all the interesting elements are of even degree, we ignore this.)

To conclude the proof, observe that for $\alpha=\gamma_{i}^{k_{0}} \times \phi_{j}^{j}$,

$$
\operatorname{res} \bar{\alpha}=\operatorname{res} \gamma_{i}^{k_{0}} \times \operatorname{res} \bar{\phi}_{j}^{j}=\sum_{i_{0}+i_{1}=i, j_{0}+j_{1}=j}\left(\gamma_{i_{0}}^{k_{0}, 0} \times \gamma_{i_{1}}^{k_{1}, 0}\right) \times\left(\phi_{j_{0}^{0}}^{j_{0}^{0}} \times \phi_{j_{1}}^{j_{1}}\right)
$$

which, after twisting to get the order right, is as required.

REMARK. The use of double cosets is perhaps misleading since they enter in two different ways which, in a sense, cancel each other. However, I find the proof given above the most natural, and it is certainly the easiest to write down.

Corollary 2. (a) $C_{i, 1}^{k}=\gamma_{i}^{k} \cdot \phi_{1}^{k}$; (b) $C_{n, k-n}^{k}=0$ for $n>0$.

Proof. (a) We have

$$
\begin{aligned}
C_{i, 1}^{k} & =\operatorname{tr}\left(\gamma_{i}^{k-1} \times \phi_{1}^{1}\right)=\operatorname{tr}\left(\left(\operatorname{res} \gamma_{i}^{k}\right) \cdot \phi_{1}^{1}\right) \\
& =\gamma_{i}^{k} \cdot \operatorname{tr}\left(1 \times \phi_{1}^{1}\right)=\gamma_{i}^{k} \cdot \phi_{1}^{k} .
\end{aligned}
$$

(See [3, Chapter XII, §8, formula (13)].)

(b) The natural representation $\pi_{i}$ of $\mathscr{S}_{i}$ is equivalent to a representation of the form $1 \oplus \rho$. (For example, $\pi_{i}$ is induced from the trivial representation of $\mathscr{S}_{i-1}$, and by Frobenius reciprocity it must contain the trivial representation as a constituent.) Thus $c\left(\pi_{i}\right)=c(1) c(\rho)=c(\rho)$, and $\gamma_{i}^{i}=c_{i}\left(\pi_{i}\right)$ $=c_{i}(\rho)=0$.

REMARK. By the above, $C_{1}=\gamma_{1}+\phi_{1}, C_{2}=\gamma_{2}+\gamma_{1} \cdot \phi_{1}+\phi_{2}$. Unfortunately, as is quite clear from the proof of Theorem 1 , the generalization of this formula to $n>2$ does not hold.

4. Applications for finite groups. In the context of $\S 2$, consider $\alpha \in H^{*}(X, Z)$ involving only even degrees. It is possible to define, in a natural way, an element $1_{S} \int_{\alpha} \in H^{*}\left(E_{S} \times{ }_{S} X^{k}, Z\right.$ ) (where $S$ is a subgroup of $\mathscr{S}_{k}$ ) whose restriction to $X^{k}$ is $\alpha^{k}$. For details of the construction and properties, see $[5, \S 3]$; compare also with [4, Chapter VII]. In particular, if we write $H^{*}\left(B_{T}, Z\right)=Z[X], \operatorname{deg} X=2$, then $1_{S} \delta(1+X)=\sum_{n} \phi_{n}^{k}$.

Let $\lambda: H \rightarrow T$ be a one-dimensional representation of a finite group $H$. Then $\lambda^{*}(X)=c_{1}(\lambda)=\lambda \in H=H^{2}(H, Z)$. Also, if $\Lambda$ is the cochain map arising from a map of $\mathrm{CW}$ complexes $B_{H} \rightarrow B_{T}$ induced by $\lambda$, then $\operatorname{Hom}\left(C_{*}\left(E_{S}\right), \Lambda^{k}\right)$ induces $\left(1 \int \lambda\right)^{*}: H^{*}\left(B_{S S T}\right) \rightarrow H^{*}\left(S \int H\right)$ (use formula (1)). Write $w_{n}^{k}(\lambda)$ for the component of $1_{\mathscr{S}_{k}} \int(1+\lambda)$ in $H^{2 n}\left(\mathscr{S}_{k} \int H, Z\right)$. Then $\quad\left(1 \int \lambda\right)^{*}\left(\phi_{n}^{k}\right)=w_{n}^{k}(1)$. Also, $\quad\left(1 \int \lambda\right)^{*}\left(\gamma_{n}^{k}\right)=\inf \left[\mathscr{S}_{k} \rightarrow \mathscr{S}_{k} \int H\right]\left(\gamma_{n}^{k}\right)$. Finally, $\left(1 \int \lambda\right)^{*}$ is clearly consistent with transfer since, if $S^{\prime}<S$, then $\left(S \int T: S^{\prime} \int T\right)=\left(S \int H: S^{\prime} \int H\right)$. Hence 
$\left(1 \int \lambda\right)^{*}\left(C_{i, j}^{k}\right)=\operatorname{tr}\left[\left(\mathscr{S}_{k-j} \times \mathscr{S}_{j}\right) \int H \rightarrow \mathscr{S}_{k} \int H\right]\left(\gamma_{i}^{k-j} \times w_{j}^{j}(\lambda)\right)$.

We have now proved

Theorem 3. Let $H$ be a finite group and $\lambda$ a one-dimensional representation of $H$. Then the Chern classes of the representation $1 \int \lambda$ of $\mathscr{S}_{k} \int H$ are given by

$$
c_{n}\left(1 \int \lambda\right)=\sum_{i+j=n} \operatorname{tr}\left[\left(\mathscr{S}_{k-j} \times \mathscr{S}_{j}\right) \int H \rightarrow \mathscr{S}_{k} \int H\right]\left\{\inf c_{i}\left(\pi_{k-j}\right) \times\left(1_{\mathscr{S}_{j}} \int \lambda\right)\right\}
$$

where $\pi_{k-j}$ is the natural representation of $\mathscr{S}_{k-i}$.

(The reader should not confuse the representation $1 \int \lambda$ with the cohomology class $1 \mathscr{S}_{j} \int \lambda=w_{j}^{j}(\lambda)$.)

REMarK. Even without the explicit formula, we may conclude some-

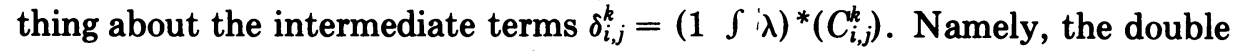
complex $\operatorname{Hom}_{\mathcal{C}}\left(C_{*}\left(E_{\mathscr{S}}\right), C^{*}(X)^{k}\right)$ yields a spectral sequence with $E_{2}$ term $H^{*}\left(\mathscr{S}, H^{*}(X)^{k}\right)$. In case $X=B_{T}$, this spectral sequence is completely trivial and reduces to the $E_{2}$ term. (Even the filtration splits.) For $X=B_{H}$ there is little we can say (with integer coefficients), but by naturality it is clear that $\delta_{i, j}^{k}$ belongs to the group $F^{2 i} H^{2(i+j)}\left(\mathscr{S}_{k} \int H, Z\right)$ of the filtration. Finally, it is not hard to see that the filtration agrees with that of the first spectral sequence of Hochschild-Serre [9] for the group extension $1 \rightarrow H^{k}$ $\rightarrow \mathscr{S}_{k} \int H \rightarrow \mathscr{S}_{k} \rightarrow 1$.

Assume next that $G$ is a finite group and $H$ a subgroup of index $k$. Let $\tau_{1}, \tau_{2}, \cdots, \tau_{k}$ be a complete set of representatives of the left cosets of $H$ in G. If $\rho \in G$, let $\rho \tau_{i}=\tau_{\bar{\rho}(i)} \sigma_{i}$ where $\bar{\rho} \in \mathscr{S}_{k}$ and $\sigma_{i} \in H$. Setting $\Phi(\rho)=\bar{\rho}$ - $\left(\sigma_{1} \times \sigma_{2} \times \cdots \times \sigma_{k}\right) \in \mathscr{S}_{k} \int H$ yields a monomorphism $\Phi: G \rightarrow \mathscr{Y}_{k} \int H$ $[5, \S 2]$. Let $\lambda$ be a one-dimensional representation of $H$; then the representation of $G$ induced from $\lambda$, $\operatorname{ind}[H \rightarrow G] \lambda$, is equivalent to $\left(1 \int \lambda\right) \circ \Phi$. Write $c_{i, j}(\lambda)=\Phi^{*}\left(\delta_{i, j}^{k}\right)$; in particular: (i) $c_{n, 0}(\lambda)=c_{n}(\pi)$ where $\pi$ is the representation obtained from the permutation representation of $G$ on the set of left cosets $G / H$ (in fact, $\pi=\operatorname{ind}[H \rightarrow G](1)$ ), and (ii) $c_{0, n}(\lambda)=\Phi^{*}\left(w_{n}^{k}(\lambda)\right.$ ) which, by definition $[5, \S 5]$, is just $\mathscr{N}_{n}(\lambda)$, the homogeneous component of $\mathscr{N}_{H \rightarrow G}(1+\lambda)$ of degree $2 n$.

Since $\Phi$ is an explicit monomorphism, we can in principle compute $c_{i, j}(\lambda)$ by means of the double coset rule for resotr [3, Chapter XII, Proposition 9.1]. Such a computation would involve determining the intersections of $\Phi(G)$ with conjugates of the subgroups $\left(\mathscr{S}_{k-j} \times \mathscr{S}_{j}\right) \int H$. Since the subgroup of $\bar{\rho} \in \mathscr{S}_{k}$ arising from $\rho \in G$ may be quite general-it need only be transitive-one would not expect the answer to be very easy in the general case.

It should also be pointed out that the methods used in this paper may be used to compute the Chern classes of the natural representations of the symmetric groups. First, by the Sylow subgroup argument, we may re- 
strict to the Sylow subgroups, and, next, by the known structure of these groups, to wreath products of the form $S \int P$ where $P$ is a cyclic permutation group of prime degree. Since the natural (regular) representation of $P$ is the direct sum of one-dimensional representations, Theorem 3 may be used to set up a computation by induction. Again, this computation is possible in principle only, but it does reduce everything to the algebraic theory of cohomology of groups.

In Theorem 4, I list those facts about the $c_{i, j}(\lambda)$ which are fairly easy consequences of the above results. In particular, notice that the situation is not completely hopeless if $H$ is normal in $G$.

THEOREM 4. (1) Let $G$ be a finite group, $H$ a subgroup, and $\lambda$ a one-dimensional representation of $H$; let $\pi=\operatorname{ind}[H \rightarrow \mathrm{G}](1)$. Then $c_{n}(\operatorname{ind}[H \rightarrow G](\lambda))$ $=\sum_{i+j=n} c_{i, j}(\lambda)$ where (a) $c_{0, n}(\lambda)=\mathscr{N}_{n}(\lambda)$, the component of $\mathscr{N}_{H \rightarrow G}(1+\lambda)$ of degree $2 n$; (b) $c_{n-1,1}(\lambda)=c_{n-1}(\pi) \cdot \operatorname{tr}[H \rightarrow G](\lambda)$; and (c) $c_{n, 0}(\lambda)=c_{n}(\pi)$. Also, (d) the highest Chern class $c_{(G: H)}($ ind $\lambda)=\mathscr{N}_{H \rightarrow G}(\lambda)$.

(2) If $H$ is normal in $G$, then (a) $c_{i, j}(\lambda) \in F^{2 i} H^{2(i+j)}(G, Z)$ of the Hochschild-Serre filtration; (b) $c_{i, j}(\lambda)=0$ if $j$ is relatively prime to the index $(G: H)$ and $i>0$; and (c) $c_{n-1,1}(\lambda)=0$ always, $(n>1)$.

Proof. If $H$ is normal in $G$, then $\Phi(H)<H^{k}$. Comparing the two group extensions and the remark following Theorem 3 make 2a clear.

To prove $2 \mathrm{~b}$, consider the intersection of $\Phi(G)$ with a conjugate of $\left(\mathscr{S}\left(K_{0}\right) \times \mathscr{S}\left(K_{1}\right)\right) \mathcal{S} H$ in $\mathscr{S}(K) \mathcal{S} H$ where $K=K_{0} \cup K_{1}$ is a partition. I claim that if $k_{1}=j$ is relatively prime to $k$, then this intersection is trivial. In fact, in the normal case, the map $\rho \rightarrow \bar{\rho}$ is simply the inflation to $G$ of the regular representation $\rho_{G / H}$ of $G / H$. Hence, if $\bar{\rho} \neq 1$, then as a permutation it is a product of disjoint cycles all of the same length dividing $k$. If $\left(k_{1}, k\right)$ $=1, \mathscr{S}\left(K_{0}\right) \times \mathscr{S}\left(K_{1}\right)$ contains no such permutations.

As above, $c_{i, j}(\lambda)$ may be computed by the double coset formula. If $(k, j)$ $=1$, all terms will involve restriction of $\left(\inf c_{i}\left(\pi_{k-j}\right)\right) \times w_{j}^{j}(\lambda)$ to $H^{k-j} \times H^{j}$ $=H^{k}$, and such restrictions are certainly 0 .

$2 \mathrm{c}$ follows from $2 \mathrm{~b}$.

Corollary 5. Let $G$ be finite and $H$ a normal subgroup of $G$ of prime index $p$; let $\lambda$ be a one-dimensional representation of $H$. Then

$$
c_{n}(\operatorname{ind} \lambda)=\mathscr{N}_{n}(\lambda), \quad \text { for } n \neq p-1,
$$

and

$$
c_{p-1}(\text { ind } \lambda)=\mathscr{N}_{p-1}(\lambda)-\inf \mu^{p-1},
$$

where $\mu$ generates $H^{2}(G / H, Z)$.

Proof.

$$
c\left(\rho_{G / H}\right)=1-\mu^{p-1} .
$$


We conclude this section with two applications of Corollary 5 to the calculation of $\mathscr{N}$.

(i) $G$ is cyclic of order $p^{a}(a>1)$ and $H$ is the subgroup of order $p^{a-1}$. Let $\chi$ be a generator of $G$ and $\chi_{0}=\operatorname{res} \chi$. Then ind $\chi_{0}=\chi \oplus \chi^{1+p^{a-1} \oplus}$ $\cdots \oplus \chi^{1+(p-1) p^{a-1}}$. Hence

$$
\begin{aligned}
c\left(\text { ind } \chi_{0}\right) & =\prod_{i=0}^{p-1}\left[1+\left(1+i p^{a-1}\right) \chi\right] \\
& = \begin{cases}(1+\chi)^{p}, & p>2 \\
1+\left(2+2^{a-1}\right) \chi+\left(1+2^{a-1}\right) \chi^{2}, & p=2\end{cases}
\end{aligned}
$$

Also

$$
\inf \mu^{p-1}=\left(p^{a-1} \chi\right)^{p-1}= \begin{cases}0, & p>2 \\ 2^{a-1} \chi, & p=2 .\end{cases}
$$

Since by $[5, \S 6], \mathscr{N}$ is multiplicative and transitive, it follows that

Proposition 5. Let $G$ be cyclic of order $p^{a}$ where $p$ is an odd prime, let $H$ be a nontrivial subgroup, and let $\chi$ generate $G$. Then

$$
\mathscr{N}_{H \rightarrow G}(\operatorname{res}(1+\chi))=(1+\chi)^{(G: H)} \text {. }
$$

(ii) $G=P \times H$ where $P$ is cyclic of prime order $p$. We have inf $\mu=\mu$ $\times 1 \in H^{2}(P \times H, Z)$. Also, if $\lambda \in H$, then ind $\lambda=\rho_{P} \times 1 \otimes 1 \times \lambda$, and

$$
\begin{aligned}
c(\text { ind } \lambda) & =\prod_{i=0}^{p-1}\left(1+1 \times \lambda+i_{\mu} \times 1\right) \\
& =(1+1 \times \lambda)^{p}-\mu^{p-1} \times(1+\lambda) .
\end{aligned}
$$

Hence

$$
\mathscr{N}_{n}(\lambda)= \begin{cases}\left(\begin{array}{l}
p \\
n
\end{array}\right) 1 \times \lambda^{n}, & n=0,1, \cdots, p-1, \\
1 \times \lambda^{p}-\mu^{p-1} \times \lambda, & n=p .\end{cases}
$$

REMARK. As observed in $[5, \S 6]$, the last line is consistent with a computation of the same quantity from the point of view of Steenrod reduced powers.

5. The dihedral group. Let $D=G p\left\{\sigma, \tau: \sigma^{4}=\tau^{2}=1, \tau^{-1} \sigma \tau=\sigma^{3}\right\}$. We may view $D$ in two ways as a split extension:

$$
\begin{aligned}
& 1 \rightarrow \Sigma=G p\{\sigma\} \rightarrow D \rightarrow D / \Sigma \rightarrow 1, \\
& 1 \rightarrow H \rightarrow D \rightarrow D / H \rightarrow 1,
\end{aligned}
$$

where $H=\left\{1, \tau \sigma, \tau \sigma^{3}, \sigma^{2}\right\} \cong Z / 2 \times Z / 2$. In each case the factor group is $Z / 2$ and acts in the obvious way on the normal (abelian) subgroup. 
By comparing the two spectral sequences of these group extensions, it is possible to compute the actual ring structure of $H^{*}(D, Z)$. It should be noted, however, that the computation seems to depend strongly on the use of the double coset formula for the map $\mathscr{N}$ [5]. (Of course, with $Z / p$ as coefficient ring, the ring structure is given by the result of Nakaoka.)

Define $\chi_{1}, \xi \in H^{2}(D, Z)=\hat{D}$ by

$$
\begin{gathered}
\chi_{1}(\tau)=1, \chi_{1}(\sigma)=-1, \\
\xi(\tau)=-1, \xi(\sigma)=1 .
\end{gathered}
$$

Define $x \in H^{2}(\Sigma, Z)=\hat{\Sigma}$ by $x(\sigma)=\sqrt{ }(-1)$. (The values are taken in the group of complex roots of unity.) Let $\chi=\mathscr{N}_{\Sigma \rightarrow D}(x)$. Then

$$
H^{*}(D, Z)=Z\left[\xi, \chi_{1}, \zeta, \chi\right]
$$

where

$$
\operatorname{deg} \xi=\operatorname{deg} \chi_{1}=2, \quad \operatorname{deg} \zeta=3, \quad \operatorname{deg} \chi=4,
$$

and

$$
\begin{aligned}
& 2 \xi=2 \chi_{1}=2 \zeta=4 \chi=0 \\
& \chi_{1}^{2}=\xi \cdot \chi_{1}, \quad \zeta^{2}=\xi \cdot \chi
\end{aligned}
$$

are the relations.

The irreducible representations of $D$ are $1, \xi, \chi_{1}, \xi \otimes \chi_{1} \in D$ and $\rho$ $=\operatorname{ind}[\Sigma \rightarrow D] x$ of degree 2 . From Theorem 4 we have

$$
c(\rho)=1+\xi+\chi .
$$

It is interesting to note in this context that the Atiyah spectral sequence [1] has only one nontrivial differentiation $d_{3}$. In fact, the generators $\xi, \chi_{1}$, $\chi$ are all Chern classes, and hence universal cycles, and

$$
d_{3} \zeta=S q^{3} \zeta \in \zeta^{2}+2 H^{6}(D, Z) .
$$

But $H^{6}(D, Z)$ is generated by elements of order 2, and hence $d_{3} \xi=\zeta^{2}$ $=\xi \cdot \chi$. Since $\xi \cdot \chi$ is not annihilated by any element of positive degree, the above contention follows. Finally, notice that what Atiyah calls $H^{\prime}(D, Z)$ is the subring generated by the Chern classes, and hence Atiyah's conjectured filtration for $R(D)$ is the correct one [1, Appendix].

\section{REFERENCES}

1. M. F. Atiyah, Characters and the cohomology of finite groups, Inst. Hautes Etudes Sci. Publ. Math. no. 9 (1961).

2. A. Borel and J.-P. Serre, Groupes de Lie et puissances réduites de Steenrod, Amer. J. Math. 75 (1953), 409-448.

3. H. Cartan and S. Eilenberg, Homological algebra, Princeton Univ. Press, Princeton, N. J., 1956. 
4. N. E. Steenrod and D. B. A. Epstein, Cohomology operations, Annals of Mathematics Studies No. 50, Princeton Univ. Press, Princeton, N. J., 1962.

5. L. Evens, A generalization of the transfer map in the cohomology of groups, Trans. Amer. Math. Soc. 108 (1963), 54-65.

6. J. Milnor, Construction of universal bundles. II, Ann. of Math. 63 (1956), 430-436.

7. M. Nakaoka, Homology of the infinite symmetric group, Ann. of Math. 73 (1961), 229-257.

8. W. Burnside, Theory of groups of finite order, Dover, New York, 1955.

9. G. P. Hochschild and J.-P. Serre, Cohomology of group extensions, Trans. Amer. Math. Soc. 74 (1953), 110-135.

\section{UNIVERSITY OF CALIFORNIA, Berkeley, California}

